\title{
Design Process of a New Lighting Robotic Arm for Operating Room
}

\author{
Juan Sandoval ${ }^{1(\bowtie)}$, Laurence Nouaille ${ }^{1}$, Gérard Poisson ${ }^{1}$, \\ Yves Parmantier ${ }^{2}$, and Benoit Magnain ${ }^{3}$ \\ ${ }^{1}$ PRISME Laboratory, INSA CVL, University of Orléans, Orléans, France \\ juan. sandoval-arevalo@univ-orleans. fr \\ 2 POLE CAPTEUR, University of Orléans, Orléans, France \\ 3 INSA CVL, Univ. Orléans, Univ. Tours, LaMé EA 7494, \\ 18022 Bourges, France
}

\begin{abstract}
In this paper, we present the design process followed to conceive a lighting robotic arm dedicated to operating rooms. During a surgical procedure, the use of a high quality lighting system is essential to the successful completion of the surgical task. Indeed, the intensity and incidence of the light projection must be controlled according to the surgeon needs. However, surgeons cannot directly manipulate the lighting dome by hand because of aseptic reasons. First of all, we present the medical specifications used to define the technological and robotic needs, provided from real experiments and observations in operating room. Then, a complete topological synthesis has been applied to choose the most adequate kinematic solution. A kinematic simulator, as well as a prototype, have been developed to validate the effectiveness of the selected solution. Finally, we propose the use of a dynamic analysis, based on a Newton-Euler algorithm, to correctly choose the joint actuators.
\end{abstract}

Keywords: Medical robots $\cdot$ Kinematics $\cdot$ Mechanism synthesis

\section{Introduction}

Nowadays, $80 \%$ of surgical interventions are still performed in conventional surgery: the surgeon makes a large incision in the patient body to easily get access to the target zone. Although the expertise and the dexterity of the surgeon is of great importance, the operating room ergonomics and environmental conditions are crucial too. For example, the mobility of the lighting system is extremely important, and poly-articulated arms supporting the lamps are used for this purpose. Typically, the lighting lamp, i.e. the dome, is fixed to the extremity of a passive poly-articulated system allowing 5 or 6 Degrees of Freedom (DoF). As depicted in Fig. 1 (left part), that mechanisms are mainly composed by a cradle (1), a hoisting arm (2) and a horizontal arm (3), respectively. In particular, the cradle is composed of two parts allowing to orientate the dome.

In order to preserve the aseptic conditions, the surgeon never touches this system during the surgical procedure. Instead, he is obligated to give orders to an assistant, situated outside of the sterile zone, to move the lamp according to his needs. Nevertheless, this communication step between the surgeon and his assistant imposes delays 
time and a loss of precision. The goal of this project is to automate the lighting control process. On this way, a camera-based system recognizes the hand surgeon gestures and traduces them in orders to move a robotized system hanging the lighting dome. Constraints linked to the positioning of the dome and to the desired light intensity on the surgical zone are the first specifications given by surgeons. Cost, ergonomic and acceptability of technical solutions are criteria provided by the industrial partner and are considered in the design process. The gestures recognition has been developed in PRISME laboratory [1-3]. In this paper we present the design process followed to obtain the final robotic solution that complies with the medical and industrial specifications.

To our knowledge, this is the first robotic solution proposed to control the position and orientation of a lighting dome in operating room. Nevertheless, medical robots with similar functionalities can be cited. SurgiScope $®$ is a ceiling mounted robotized platform used in neurosurgery, carrying a microscope with a mass of around $40 \mathrm{~kg}$ and based on a delta parallel structure [4]. It is commercialized by the ISIS company. The Automated Endoscopic System for Optimal Positioning robot (ASEOP), used for laparoscopic surgery [5], consists of an endoscope carrier arm controlled by the surgeon's voice.

In the first part of this paper we briefly describe the proposed design process. The medical specifications are described in Sect. 3. We develop in Sect. 4 the topological synthesis phase allowing to find the appropriate kinematic structure. Section 5 reports a description of the simulator developed to validate the kinematic solution. Section 6 presents the dimensional analysis used to select the adapted actuators. Conclusions about the effectiveness of the kinematic solution and the proposed design process are presented in the last section.
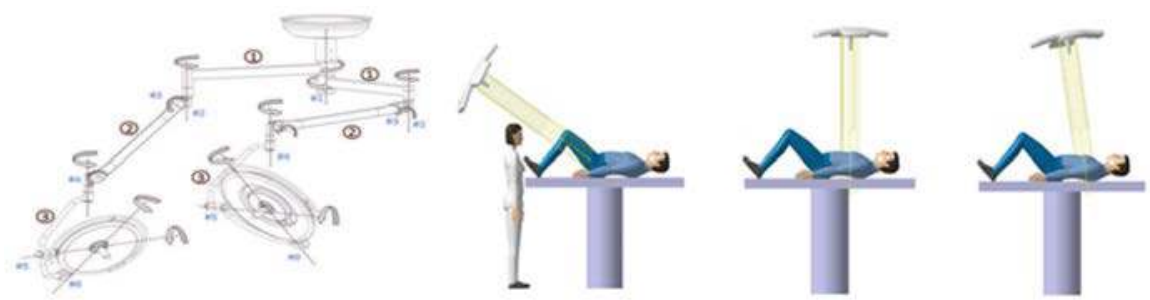

Fig. 1. (left) Kinematics of standard lighting arm for operating room; (right) typical positions of the lighting dome in an operating scene

\section{Adopted Design Process}

Adapted from [6], the design process used to design the robotized arm is depicted in Fig. 2. The first step concerns the analysis and characterization of the medical gestures, as the ones shown in Fig. 1 (right). The second step is the topological synthesis: based on the medical specifications including the results of the first step, several kinematic solutions were proposed. In the third step we choose the most appropriate kinematic solution: the proposed solutions are evaluated and compared based on identified criteria 
through a decisional matrix. The last step concerns the dimensional synthesis of the selected solution, including the selection of actuators performed through a dynamic analysis based on the Newton-Euler method.

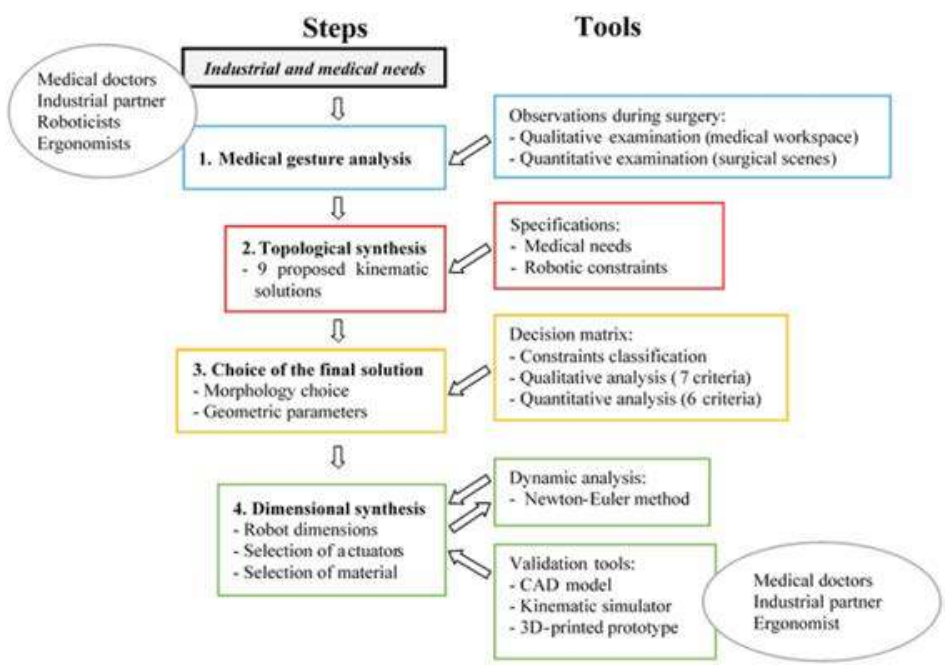

Fig. 2. Design process carried out

\section{Analysis and Medical Gesture Characterization}

The industrial partner of the project, Maquet SAS, defined the specifications for the design of the robotized system, based on observations made during surgical procedures.

From these observations, three scenes are pointed (Fig. 1 - right): in a first scene, the lighting dome is situated behind the surgeon's head; in a second one, a vertical projection of the beams of light towards the patient becomes necessary and in the last one, the dome must turn around a fixed point considered like a remote center of motion (RCM). Moreover, the study allowed to define three different medical cases of displacement for the dome [7]. According to these cases, the solution should allow movements of the lighting dome in the horizontal plane as well as to turn around the three Cartesian axis.

\section{Topological Synthesis}

After studying the specifications and the criteria defined by the company, the topological synthesis is performed to propose kinematic structures corresponding to the medical needs. First, several kinematic structures corresponding to the expressed needs are proposed and then evaluated. A multi criteria analysis using a decision matrix, allowing to include several criteria, is carried out. This step gives us the most appropriate structure to the expected device. 


\subsection{Considered Kinematic Structures}

With the purpose of guaranteeing the specifications defined by our industrial partner, the robotic solution would need at least $4 \mathrm{DoF}$, concerning the translation along the horizontal plane $(2 \mathrm{DoF})$ and the orientation of the lighting dome around the axis of the horizontal plane (2 DoF). Nevertheless, the manual existing arm has only 3 DoF. Nine kinematic structures have been identified, as shown in Table 1 . Solutions with only 3 DoF are proposed, these solutions limit the robot movements but are advantageous in terms of lightweight of the structure and a lower production cost. Two parallel structures are also proposed. A parallel robot is usually more complex in terms of control than a serial one, but it offers a better mechanical balancing, a great accuracy and it can perform faster movements than serial manipulators.

Table 1. Proposed kinematic solutions

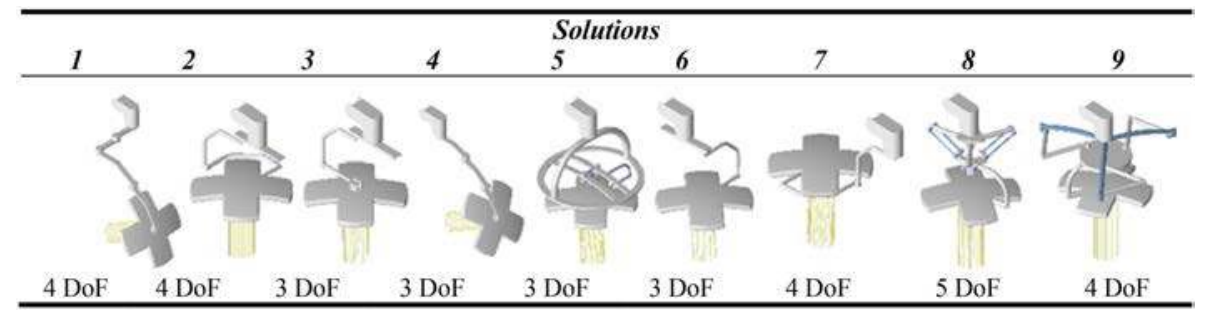

Although high velocities are not required for our application, the control of the mechanical balancing is a point of great importance. Nevertheless, solutions with serial kinematics have better workspace/compactness ratio than parallel solutions, which is a key point for our application.

\subsection{Evaluation Process}

First, 6 criteria considering the specifications are defined to evaluate the proposed solutions: maximum displacement of the center of mass $(\mathrm{CoM})$ of the dome in the plane, maximum rotation of the dome around the two directions of the horizontal plane and around the vertical axis, number of links, at least one posture must allow the coincidence between the CoM and the first vertical axis, the supporting link designed by the company can attach the solution and the solution must not disturb the lighting functionalities. There are three grade possibilities: if the criterion is always accomplished by the robot, the given grade is "Yes", in the case of certain conditions are required to accomplish the criterion we assign the grade "Possible", and finally if the criterion is never accomplished then the grade "Not possible" is assigned. If the last grade is given, the solution is then penalized for the next part of the decision matrix. Moreover, each criterion has an assigned flexibility level, i.e. degree of priority. For instance, once a criterion with flexibility "none" gets a grade "Not possible", the solution is removed. 
In a second step, 8 performance index were defined to classify the 9 kinematic solutions: number of DoF lost in case of motor failure, possibility to control the vertical translation and along the horizontal plane of the lighting dome, number of links, number of Dof, similarity with the existent non-robotized arm, types of joints, aseptic features and kinematic complexity. A weight is attributed to each performance index to define a priority order between the indexes. Thus, the kinematic solutions are evaluated by assigning a grade between 0 and 1 for each criterion. Each weight-grade product is calculated and these products are added to obtain the total score of each solution, as presented in Fig. 3. From these results, the solution $\mathrm{N}^{\circ} 7$ is defined as the optimal kinematic solution. The dimensional phase of the selected solution is presented in the next section.

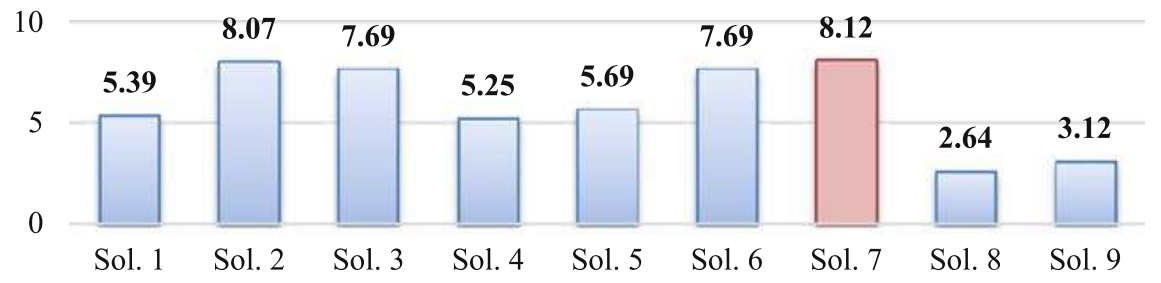

Fig. 3. Classification of nine kinematic solutions

\section{Kinematic Simulator}

In order to allow a functional validation of the selected solution from the surgeon point of view, a simulator has been developped using $\mathrm{C}++$ programming langage and the OPENGL library (Fig. 4). Two ways are proposed to manipulate to robot: using the keyboard or through the hand gestures.

According to the specifications provided in Sect. 3, the kinematic solution should permit the movement of the lighting dome in the horizontal plane (denoted X-Y) as well as the rotation around the $\mathrm{X}$ and $\mathrm{Y}$ axis. On this way, we adapted the interface to mainly implement three functionalities, allowings to control the lighting dome movements in this horizontal plane, the rotation around the $\mathrm{X}$ and $\mathrm{Y}$ axis, and the graduation of the light intensity. A second mode was also implemented, allowing a joint decoupled position control, which could be used in case of emergencies but that seems less useful for the surgeons. Different performed movements are shown in Fig. 4, evidencing that the selected solution effectively meets the specifications imposed in Sect. 3. 


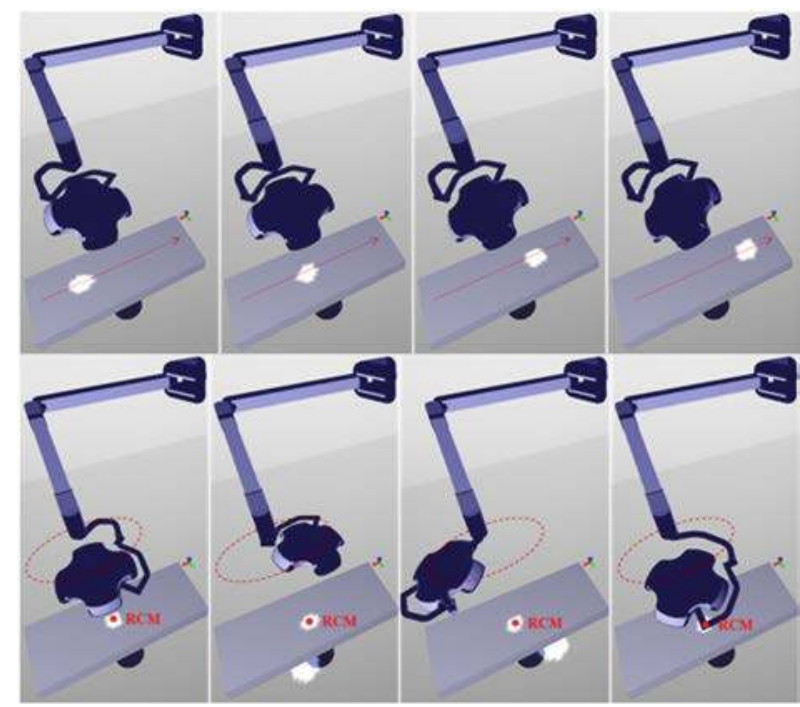

Fig. 4. Virtual simulator screenshoots

\section{Dimensional Synthesis Using Dynamics Analysis}

As resumed in Fig. 5, a dynamic analysis is carried out for the designated solution, allowing to calculate the motor characteristics of each joint and the bending/torsional forces suffered by each link. The Newton-Euler algorithm [8] was adapted here, considering the maximum joint velocities and accelerations, in order to take into account the inertial effects. Moreover, the algorithm applied allows to find the maximum motor torques, evaluating the overall joint state combinations.

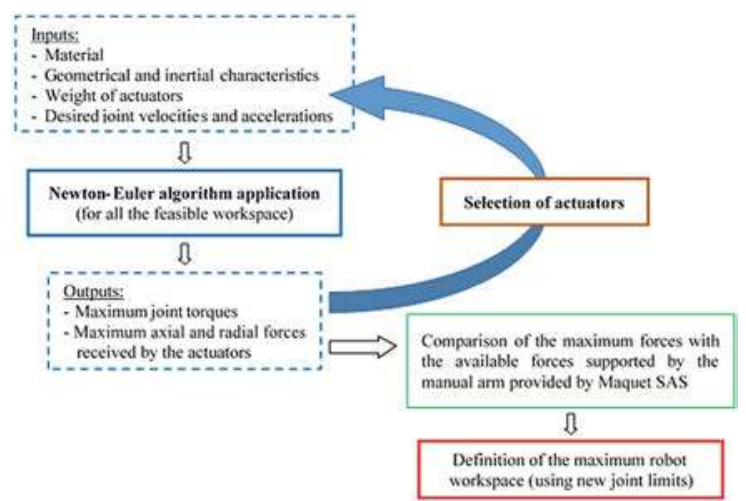

Fig. 5. Algorithm proposed to select the joint actuators and to define a feasible robot workspace 
Solution $\mathrm{N}^{\circ} 7$ has $4 \mathrm{DoF}$, concerning 2 DoF allowing the translation of the dome in the horizontal plane and $2 \mathrm{DoF}$ for the orientation of the dome. Moreover, the solution is composed of 4 revolute joints $\left(q_{4}, q_{5}, q_{6}, q_{7}\right)$, as shown in Fig. 6. The material considered is an aluminum alloy $\left(\rho=2710 \mathrm{~kg} \cdot \mathrm{m}^{-3}\right)$ for links S4 to S6 and a plastic $\left(\rho=1050 \mathrm{~kg} \cdot \mathrm{m}^{-3}\right)$ for the lighting dome S7. The section of each link is tubular $\left(\varnothing_{\text {out }}=50 \mathrm{~mm}, \varnothing_{\text {in }}=45 \mathrm{~mm}\right)$. Thus, the weight of links S4, S5 and S6 are $0.926 \mathrm{~kg}, 0.351 \mathrm{~kg}$ and $1.094 \mathrm{~kg}$, respectively. The weight of the lighting dome is $20.16 \mathrm{~kg}$. Moreover, a maximum joint velocity $\dot{q}_{i}=\pi / 32 \mathrm{rad} \cdot \mathrm{s}^{-1}$ and acceleration $\ddot{q}_{i}=\pi / 32 \mathrm{rad} \cdot \mathrm{s}^{-2}$ for $i=4$ to 7 were used.
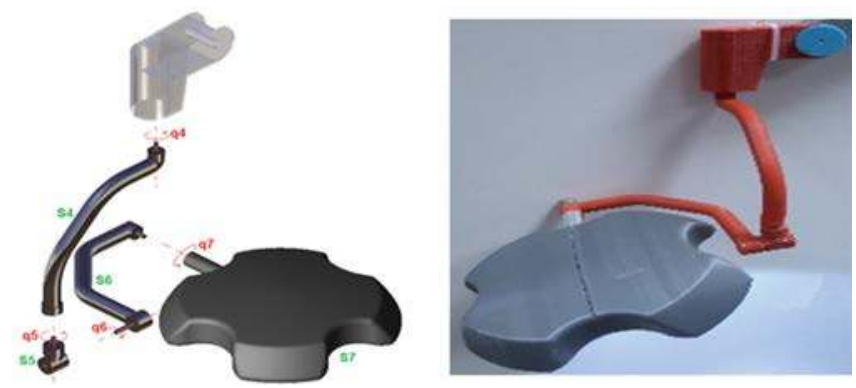

Fig. 6. Kinematic design of solution 7 (left). 3D-printed prototype of Solution $\mathrm{N}^{\circ} 7$ (right)

Newton-Euler algorithm is applied in two different steps for all the joints, taking into account the following joint limits: $q_{4}=0^{\circ}, 0^{\circ} \leq q_{5} \leq 31^{\circ},-90^{\circ} \leq q_{6} \leq 90^{\circ}$ and $-90^{\circ} \leq q_{7} \leq 90^{\circ}$. The first obtained results don't take into account motor weights because they are not chosen yet. We choose the motors and apply the algorithm again, including the motor weights. If the motor torques and axial forces exceed the motor capabilities, other different actuators must be determined. Table 2 summarizes the obtained results for the two steps of calculation. In order to have a real vision of the mechanism, a 3D printed prototype with a model reduced to a quarter scale was built (Fig. 6 - right). The presented design method for a lighting robotic arm dedicated to operating rooms allowed us to find an innovative solution. This kinematic solution has been protected by a patent [9].

Table 2. Results obtained in the first step (without motor weights)/in the second step (with motor)

\begin{tabular}{l|l|l|l|l}
\hline & Joint 4 & Joint 5 & Joint 6 & Joint 7 \\
\hline Maximum Radial force [N] & 1.2356 & 1.2464 & 208.33 & $197.60 / 203.14$ \\
\hline Maximum Axial force [N] & $220.85 / 242.99$ & $211.77 / 228.38$ & 0.82 & $197.61 / 203.14$ \\
\hline $\begin{array}{l}\text { Maximum Bending } \\
\text { moment [Nm] }\end{array}$ & $46.86 / 49.51$ & $85.79 / 88.37$ & $73.73 / 75.66$ & 69.98 \\
\hline Maximum Motor torque [Nm] & 0.49 & 0.61 & 4.44 & 3.71 \\
\hline
\end{tabular}




\section{Conclusions}

In this paper, we have presented the process applied to design a robotized lighting system for operating rooms. The presented work was developed in the context of a regional project, in association with different industrial, medical and ergonomist partners. According to the specifications of the particular application, we proposed and evaluated several kinematic solutions, by using a multi-criteria method. The solution obtaining the best score was completely defined in a dimensional synthesis phase using a Newton Euler's method. Moreover, we designed a virtual simulator allowing to the medical experts to validate the effectiveness of the selected solution. The presented device has been patented [9], in order to protect the kinematic design of this robotized lighting arm for operating room. Moreover, a scaled 3D printed prototype was built. The future work concerns the building of a pre-industrial prototype, in order to make first experiments on animals before proceed to a commercial phase.

Acknowledgments. This research was supported by the Région Centre-Val de Loire (France), in the context of a project named SMILE, meaning Sterile Manipulating Interface for Lighting Equipment.

\section{References}

1. Collumeau, J.-F., et al.: Hand-gesture recognition: comparative study of global, semi-local and local approaches. In: 7th International Symposium on Image and Signal Processing and Analysis (ISPA), Zagreb, Croatia, pp. 247-252 (2011)

2. Collumeau, J.-F., et al.: Hand gesture recognition using a dedicated geometric descriptor. In: 3rd International Conference on Image Processing Theory, Tools and Applications (IPTA), Istanbul, Turkey, pp. 287-292 (2012)

3. Collumeau, J.-F., et al.: Simulation interface for gesture-based remote control of a surgical lighting arm. In: IEEE International Conference on Systems, Man, and Cybernetics, Cardiff, United Kingdom, pp. 4670-4675 (2013)

4. Clavel, R.: Device for movement and displacing of an element in space. US Patent $\mathrm{n}^{\circ}$ 4976582, date of patent: 11 December 1990

5. Allaf, M.E., et al.: Laparoscopic visual field: voice versus foot pedal interfaces for control of the AESOP robot. Surg. Endosc. 12, 1415-1418 (1998)

6. Nouaille, L., et al.: Design process for robotic medical tool guidance manipulators. Proc. Inst. Mech. Eng. Part C J. Mech. Eng. Sci. 230(2), 259-275 (2015)

7. Sandoval, J., Nouaille, L., Poisson, G., Parmantier, Y.: Kinematic design of a lighting robotic arm for operating room. In: Zeghloul, S., Romdhane, L., Laribi, M. (eds.) Computational Kinematics. Mechanisms and Machine Science, vol. 50. Springer, Cham (2018)

8. Craig, J.: Introduction to Robotics: Mechanics and Control, 2nd edn. Addison-Wesley Longman Publishing Co., Inc., Boston (1989)

9. Poisson, G., et al.: Suspension de plafond à cinématique améliorée pour porter une pièce d'équipement. In: Institut National de la propriété intellectuelle, France, patent application $\mathrm{N}^{\circ}$ BR-27301/FR (2016) 